\title{
Longitudinal diet quality is not associated with depressive symptoms in a cohort of middle-aged Australian women
}

\author{
Jun S. Lai ${ }^{1 *}$, Christopher Oldmeadow ${ }^{2}$, Alexis J. Hure ${ }^{1,2}$, Mark McEvoy ${ }^{1,2,3}$, Julie Byles ${ }^{1,2,4}$ and \\ John Attia ${ }^{1,2,3,5}$ \\ ${ }^{1}$ School of Medicine and Public Health, University of Newcastle, Callaghan, NSW 2308, Australia \\ ${ }^{2}$ Hunter Medical Research Institute, New Lambton, NSW 2305, Australia \\ ${ }^{3}$ Centre for Clinical Epidemiology and Biostatistics, University of Newcastle, Callaghan, NSW 2308, Australia \\ ${ }^{4}$ Research Centre for Gender, Health and Ageing, University of Newcastle, Callaghan, NSW 2308, Australia \\ 5John Hunter Hospital, New Lambton, NSW 2305, Australia \\ (Submitted 14 May 2015 - Final revision received 25 November 2015 - Accepted 4 December 2015)
}

\section{Abstract}

There is increasing evidence for the role of nutrition in the prevention of depression. This study aims to describe changes in diet quality over 12 years among participants in the Australian Longitudinal Study on Women's Health in relation to changes in depressive symptoms. Women born between 1946 and 1951 were followed-up for 12 years (2001-2013). Dietary intake was assessed using the Dietary Questionnaire for Epidemiological Studies (version 2) in 2001, 2007 and every 2-3 years after that until 2013. Diet quality was summarised using the Australian Recommended Food Score (ARFS). Depressive symptoms were measured using the ten-item Centre for Epidemiologic Depression Scale at every 2-3-year intervals during 2001-2013. Linear mixed models were used to examine trends in diet quality and its sub-components. The same model including time-varying covariates was used to examine associations between diet quality and depressive symptoms adjusting for confounders. Sensitivity analyses were carried out using the Mediterranean dietary pattern (MDP) index to assess diet quality. Minimal changes in overall diet quality and its sub-components over 12 years were observed. There was a significant association between baseline diet quality and depression $(\beta=-0 \cdot 24, P=0 \cdot 001)$, but this was lost when time-varying covariates were added $(\beta=-0 \cdot 04, P=0 \cdot 10)$. Sensitivity analyses showed similar performance for both ARFS and MDP in predicting depressive symptoms. In conclusion, initial associations seen when using baseline measures of diet quality and depressive symptoms disappear when using methods that handle time-varying covariates, suggesting that previous studies indicating a relationship between diet and depression may have been affected by residual confounding.

\section{Key words: Diet trends: Diet quality: Depressive symptoms: Longitudinal studies: Females}

Depression is a common mental health disorder, which can lead to severe disability and poor quality of life ${ }^{(1)}$. Current interventions used to manage depression include both psychological interventions and medications, but they are only partially adequate in reducing symptom burden, with medications showing minimal benefit in sub-threshold depression ${ }^{(2)}$ and psychological interventions only reducing the incidence rate by $20-25 \%{ }^{(3)}$. Considering the high burden of this disorder, preventive strategies are also needed to reduce the public health consequences and costs. A multidisciplinary approach to depression prevention is important to reflect the multiple factors affecting the development and course of depression ${ }^{(4)}$, with particular attention given to modifiable behaviours such as diet that can potentially prevent this disorder.

In recent years, research into the relationship between dietary patterns and the risk of depression has expanded ${ }^{(5,6)}$.
There are two main approaches to defining dietary patterns: the use of statistical exploratory methods derived from reported dietary intakes and the use of dietary scores or indices ${ }^{(7)}$. The use of dietary scores or indices may be more useful in public health practice as it allows the assessment of the population's adherence to current dietary recommendations based on empirical evidence ${ }^{(8)}$.

It is also important to consider how dietary patterns may change for individuals and populations over time to guide future health promotion policy. There is evidence suggesting that the dietary intakes of Australians have changed in the past decade. In particular, cross-sectional surveys of the Australian population showed reductions in the overall per cent energy from sugar and $\mathrm{SFA}^{(9)}$. A number of cohort studies examining longitudinal changes in dietary intakes have been reported. The Blue Mountain Eye Study found an increase in intakes

Abbreviations: ARFS, Australian Recommended Food Score; DQES v2, Dietary Questionnaire for Epidemiological Studies version 2; MDP, Mediterranean dietary pattern.

*Corresponding author: J. S. Lai, email jun.lai@uon.edu.au 
of $n-3$ fatty acid and fish but also a decrease in wholemeal/grain bread consumption from 1992 to 2004 among older adults living in Sydney, Australia ${ }^{(10)}$. The Nambour Skin Cancer Study found an overall improvement in diet quality from 1992 to 2007 among residents of Queensland ${ }^{(11)}$. Despite the long-standing recognition that a variety of dietary dimensions is important for the prevention of chronic diseases, a limited number of studies have explored trends in overall diet quality in relation to chronic diseases.

Monitoring dietary trends and their association with chronic diseases in middle-aged women is of considerable interest, because this group of individuals has a higher prevalence of chronic diseases compared with their younger counterparts ${ }^{(12)}$. Although depression prevalence decreases with age, episodes experienced later in life are more likely to persist if untreated ${ }^{(13)}$. Prognosis of depression also deteriorates with age mainly because of the presence of co-morbidities and the many cases of depression that go undiagnosed because of that. Furthermore, women are twice as likely as men to be diagnosed with depression $^{(12)}$.

Previously, we have shown that maintaining good diet quality over a 6-year period is associated with reduced odds of incident depression among middle-aged women participating in the Australian Longitudinal Study on Women's Health (ALSWH) ${ }^{(14)}$. This previous study examined the diet-depression link from a prevention perspective, whereas the present analyses aimed to explore whether good diet quality relieves existing depressive symptoms, making it a potential therapeutic strategy for depression. Evidence from prospective studies on a healthy diet in effectively relieving existing depressive symptoms is scarce. Furthermore, we recognised that depression is often characterised by fluctuating symptom levels over time instead of a continuum of severity ${ }^{(15)}$. Therefore, in this study, we aim to (1) describe changes in diet quality over 12 years in the same group of women and (2) examine how a change in diet relates to depressive symptoms longitudinally, whereby depressive symptoms are treated as a continuous variable rather than as a categorical variable (i.e. with or without depression).

\section{Methods}

\section{Study sample}

The ALSWH is an ongoing prospective cohort study of over 50000 Australian women from four age cohorts - women born during 1989-1995, 1973-1978, 1946-1951 and 1921-1926(16,17). A total of 40392 women from the 1973-1978 cohort, 1946-1951 cohort and 1921-1926 cohort were recruited in 1996 and were followed-up for almost 20 years $^{(16)}$. In 2012, a new cohort of 17069 women born during 1989-1995 was recruited ${ }^{(17)}$. Women from these age cohorts were randomly selected from the Medicare health insurance database, which includes all Australian citizens and permanent residents, with overrepresentation of women living in rural and remote areas. The respondents have been shown to be broadly representative of the national population of women at baseline. Further details of this study have been described elsewhere ${ }^{(16,17)}$. This research was conducted according to the guidelines laid down in the

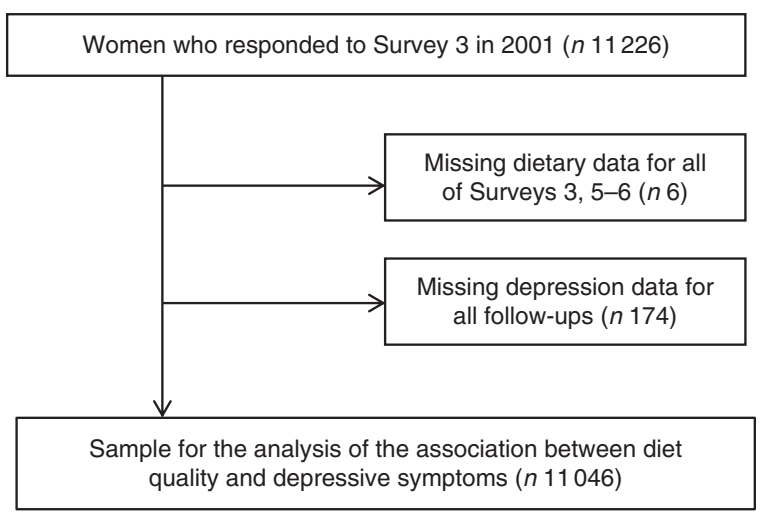

Fig. 1. Study sample selection flow diagram for analysis of the association between diet quality and depressive symptoms among women in the 1946-1951 cohort of the Australian Longitudinal Study on Women's Health.

Declaration of Helsinki, and all procedures were approved by the Human Research Ethics Committees of the University of Newcastle and the University of Queensland, Australia. Written informed consent was obtained from all participants before inclusion to the study.

In line with the rationale of our study, our sample was obtained from the 1946-1951 cohort. Women aged 45-50 years completed a self-administered questionnaire at the baseline survey in 1996 (Survey 1) and at each follow-up at approximately 3-year intervals. Studying the characteristics of women from this cohort will provide useful perspectives on the profile of depression and dietary behaviour for this middle-aged group. For this study, only data from Surveys 3-7 (2001-2013) were used, because dietary information was included in the questionnaires starting in 2001. A total of 11226 women responded to Survey 3 in 2001. The retention rate was high at each survey cycle, and $>80 \%$ of the initial study sample remained at Survey 7 in 2013. Participants were included in the present study if they had data on dietary intakes for at least one of Surveys 3, 5 or 6 as well as depressive symptoms during any subsequent follow-ups (Fig. 1); these statistical analyses included a total of 11046 women.

\section{Dietary intake}

Dietary intake was first measured at Survey 3 (2001) and then at Surveys 5, 6 and 7 (in 2007, 2010 and 2013). At Surveys 3 and 7, the Dietary Questionnaire for Epidemiological Studies version 2 (DQES v2) ${ }^{(18)}$ was administered to all participants. This questionnaire was developed by the Cancer Council Victoria for assessing dietary intakes among Australian adults in epidemiological studies. The DQES v2 requires participants to report their usual consumption over the past 12 months of seventy-four foods using a ten-point frequency scale ranging from 'never' to 'three or more times per day' and six types of alcoholic beverages with options ranging from 'never' to 'every day'. In addition, there are ten questions on the amount of fruit, different types of vegetables, milk, bread, sugar and eggs consumed and on the type of milk, bread, fat spreads and cheese used. Further details of the DQES v2 are presented elsewhere $^{(18)}$. The performance of the DQES v2 has been evaluated in multiple studies against weighed food records, and 
correlation coefficients for most nutrient intakes were comparable with those reported for other $\mathrm{FFQ}^{(19,20)}$.

A shortened version of the DQES v2 was administered to participants at Surveys 5 and 6 . This version assesses the consumption of sixty-eight foods instead of the seventy-four foods in the original version. A shortened frequency scale was also used for the majority of food items ('never', 'less than once a week' or 'once a week or more'), except for dairy products, meat and fish, which were assessed with five frequency response options ranging from 'never' to 'five or more times per week'. Nine questions on the amount of fruit, different types of vegetables, milk, bread, sugar and eggs consumed and the type of milk, bread, fat spreads and cheese used were retained. The question on the amount of sugar consumed per day was removed, and a question on the number of servings of vegetables consumed per day was included in its place. The shortened DQES v2 was designed to minimise participant burden while still facilitating a summary of diet quality, using the Australian Recommended Food Score (ARFS) method (described below).

\section{Diet quality}

Diet quality scores were calculated based on DQES v2 items using the ARFS method by Collins et al. ${ }^{(21)}$, which was modelled based on the Recommended Food Score by Kant \& Thompson ${ }^{(22)}$. The ARFS was previously validated using the ALWSH cohort and provides reasonable rankings for middleaged women according to their diet quality and nutrient intake $^{(21)}$. The scoring method assigns points for the consumption of desirable foods at the recommended levels, consistent with national recommendations in the Dietary Guidelines for Australian Adults ${ }^{(23)}$ and the core foods given in the Australian Guide to Healthy Eating ${ }^{(24)}$. Desirable food items consumed at least once a week were assigned 1 point ${ }^{(21)}$. For questions assessing the type and amount of core foods, points were assigned for consuming at least two servings of fruit a day, at least four servings of vegetables per day, at least four slices of bread per day, use of PUFA or MUFA spreads or no-fat spread, use of low-fat dairy products and consuming at least $500 \mathrm{ml}$ of milk/d. In addition, a maximum of 2 points was assigned for alcohol consumption: 1 point for frequency (up to $4 \mathrm{~d} /$ week) and 1 point for quantity (not more than two drinks on days when alcohol was consumed). The maximum ARFS score is 74 , with higher values corresponding to a healthier diet. There are seven sub-components to the ARFS: vegetables (22 points), fruits (14 points), protein foods (14 points), grains (14 points), dairy products ( 7 points), fats ( 1 point) and alcohol ( 2 points). Missing values were recoded to 0 for up to four items. Participants with greater than four missing values were considered as having incomplete data. The scores were calculated in the same manner for the shortened version of the DQES v2 used in Surveys 5 and 6. Further details on the scoring system are described elsewhere ${ }^{(21)}$.

\section{Depressive symptoms}

Participants were asked to complete the ten-item Centre for Epidemiologic Studies Depression (CES-D) scale assessing depressive symptoms during the past week at each survey. Each item was rated on a four-point scale, ranging from 'none of the time' to 'most of the time ${ }^{,(25)}$. Possible scores range from 0 to 30 , where higher scores indicate greater severity of depressed mood. The ten-item CES-D has high levels of reliability and validity against a set of reference measures assessing life satisfaction, self-rated health and social support ${ }^{(26)}$, and it produces results consistent with those measured using the twenty-item version ${ }^{(25)}$

\section{Covariates}

A number of potential confounding variables were considered. Socio-demographic variables included area of residence (urban, rural and remote), marital status (married/de facto, separated/ divorced, widowed, never married), average household income (\$0-25999 annually, \$26000-51999 annually, \$52 000 or more annually) and education status (no formal education/school certificate, higher school or trade certificate/diploma, university degree). Health behaviours were smoking status (never smoked/former smoker, light smoker, heavy smoker) and physical activity, measured using minutes of metabolic equivalents of task (MET, min) based on self-reported walking, and moderate and strenuous physical activity (nil/sedentary, 0-40 MET-min/week; low, 40-600 MET-min/week; moderate, 6001200 MET-min/week; high $\geq 1200$ MET-min/week). Self-reported physician diagnosis of depression and use of antidepressants were also included as potential confounders. These variables were reported at all surveys, except that education was obtained from Survey 1 (1996), average income from Survey 3 (2001) and information on the use of antidepressants was obtained from data linkage with the Pharmaceutical Benefit Scheme database.

\section{Statistical analyses}

Characteristics of the participants at Survey 3 (2001) according to quintiles of ARFS were compared using the $\chi^{2}$ test. A linear mixed model was used to examine changes in diet quality between 2001 and 2013. Diet quality (ARFS) at each survey was the outcome variable, and year of observation was included in the model as a fixed effect and a random subject-specific intercept to account for serial correlations with a compound symmetric variance-covariance error structure. The estimated coefficient for year corresponded to the yearly change in diet score. The analysis was repeated for each sub-component of ARFS: vegetables, fruit, protein foods, grains, dairy products, fats and alcohol.

A similar model was used to examine the longitudinal association between ARFS and depressive symptoms. A theoretical model in the form of a directed acyclic graph was set up, in which diet quality at one survey predicts change in depressive symptoms at the following survey (online Supplementary Fig. S1). Depressive symptoms at each survey were treated as the continuous outcome variable, and ARFS was categorised into quintiles and included in the model as a lagged time-varying variable. The models included adjustment for all potential confounders described above. Smoking status and physical activity were coded as time-varying categorical variables, 
whereas the remaining variables were considered as timeindependent variables. A survey-wave indicator was also included to explicitly model any secular trends in depressive symptoms.

We used all the available data from each participant under the missing-at-random assumption. All the analyses were carried out using Stata, version 13 (StataCorp LP). The significance level was set at $P<0 \cdot 05$.

\section{Sensitivity analyses}

The analyses examining the association between diet quality and depressive symptoms were repeated using diet quality scores calculated using the Mediterranean dietary pattern (MDP) index by Trichopoulou et $a l{ }^{(27)}$. Considering the fact that diet quality indices have not been tested extensively in their ability to predict the risk of depression ${ }^{(28)}$, comparing results from multiple indices can help ascertain the robustness of our study findings. Further details of the scoring method are described elsewhere ${ }^{(27)}$. In brief, the MDP index assigns a score of 0 or 1 based on daily intake of nine components. One point was assigned if the participant's intake was over the sample median for each of the following: ratio of MUFA:SFA intake, legumes, cereal, fruit and nuts, vegetables and fish. Participants received 1 point if intake was below the sample median for meat and meat products and dairy products. For the alcohol component, 1 point was scored if consumption was $5-25 \mathrm{~g} / \mathrm{d}$. The scores were categorised into five quintiles ( $0-2,3,4,5$ and $6-9$ points). The MDP index could only be calculated for Surveys 3 and 7 when the full version of DQES v2 was used; results from this analysis will only be compared with that of the ARFS at the two surveys. All the variables in the sensitivity analyses were treated as time-independent variables because of having data only at two time points.

\section{Results}

Characteristics of participants by quintiles of ARFS are presented in Table 1 . Women with higher diet quality scores were more likely to be married or in a de facto relationship, have higher household income, have higher educational status, to be physically active and less likely to smoke.

\section{Trends in diet quality 2001-2013}

Total diet quality was found to remain stable throughout the follow-up period (Table 2). Similarly, the score for the fruit component did not change significantly over time. There were significant decreasing trends in scores for vegetables, grains and fats components. For protein foods, dairy products and alcohol components, significant upward trends in scores were observed. However, these changes were negligible $(\beta= \pm 0 \cdot 05$; $95 \% \mathrm{CI} \pm 0 \cdot 01,0 \cdot 06$ ).

\section{Diet quality and depressive symptoms}

The initial univariate model showed a significant association between diet quality and depressive symptoms $(\beta=-0 \cdot 13$, $\left.P_{\text {trend }}=0.001\right)$. There was a gradual reduction in depressive symptoms across quintiles of diet quality with the highest quintile showing the greatest reduction in depressive symptoms (Table 3). Further adjustment for indicators of socio-economic status (model 2) attenuated the association observed, and adjustments for health behaviours (model 3) reduced the magnitude of the association even further. The final adjustment for self-reported physician diagnosis of depression and use of antidepressants (model 4) did not change the study estimates and significance level substantially. There were no significant associations between each sub-component of the diet quality score and depressive symptoms (data not shown).

\section{Sensitivity analyses}

When analyses were repeated using ARFS and MDP scores at Surveys 3 and 7, a significant inverse association between diet quality and depressive symptoms was observed, after adjusting for all potential confounders (Table 4). Diet quality assessed using the ARFS $\left(\beta=-0 \cdot 20, P_{\text {trend }}=0 \cdot 001\right)$ produced a greater reduction in depressive symptoms compared with the MDP index $\left(\beta=-0 \cdot 08, P_{\text {trend }}=0 \cdot 007\right)$. To determine whether this was a result of using time-independent variables instead of time-varying variables, we repeated the model using ARFS at Surveys 3 and Surveys 5-7 coding all variables as timeindependent variables. Contrary to the results in Table 3, a significant inverse association between ARFS and depressive symptoms remained $\left(\beta=-0 \cdot 24, P_{\text {trend }}=0 \cdot 001\right)$ after adjusting for all potential confounders (online Supplementary Table S1).

\section{Discussion}

This study described the trends in diet quality over a period of 12 years, and examined how these changes relate to depressive symptoms longitudinally. The results suggest that overall diet quality among this group of middle-aged women remained stable throughout the follow-up period. Although statistical tests revealed significant temporal trends in the sub-components of diet quality score, the changes in score were minimal $(<0 \cdot 1$ point change/year). These findings are similar to those in the study by Arabshahi et $a l^{(11)}$, reporting an overall improvement in diet quality among a sample of Australian adults but found that women in the older age group ( $\geq 45$ years) showed minimal improvement in diet quality than their younger counterparts.

We did not observe a significant association between diet quality and depressive symptoms, contrary to findings from two other cohort studies that evaluated the association using dietary indices $^{(29,30)}$. The previous two studies used the Alternative Healthy Eating Index and MDP index to assess diet quality, which have been constructed differently to the ARFS and have different scoring methods. To allow direct comparison, we repeated the analyses using the MDP index to assess diet quality. Although we were only able to compare results for ARFS and MDP index at two time points and treat all variables as time-independent variables, the results suggest that both scores affect depressive symptoms in a similar direction (inverse association) with ARFS resulting in greater reduction in depressive symptoms. 
Table 1. Participant characteristics in 2001 by diet quality quintiles (Q) for women in the 1946-1951 cohort of the Australian Longitudinal Study on Women's Health ( $n$ 11 046) (Mean values and standard deviations; numbers and percentages)

\begin{tabular}{|c|c|c|c|c|c|c|c|c|c|c|c|}
\hline \multirow[b]{3}{*}{ Characteristics in $2001^{*}$} & \multicolumn{10}{|c|}{$\mathrm{Q}$ of diet quality } & \multirow[b]{3}{*}{$P$} \\
\hline & \multicolumn{2}{|c|}{ Q1 } & \multicolumn{2}{|c|}{ Q2 } & \multicolumn{2}{|c|}{ Q3 } & \multicolumn{2}{|c|}{ Q4 } & \multicolumn{2}{|c|}{ Q5 } & \\
\hline & Mean & SD & Mean & SD & Mean & SD & Mean & SD & Mean & SD & \\
\hline Diet quality† & $\begin{array}{l}19 \cdot 6 \\
n\end{array}$ & $\begin{array}{l}4.2 \\
\%\end{array}$ & $\begin{array}{l}27 \cdot 7 \\
n\end{array}$ & $\begin{array}{l}1.6 \\
\%\end{array}$ & $\begin{array}{l}32 \cdot 4 \\
n\end{array}$ & $\begin{array}{l}1.3 \\
\%\end{array}$ & $\begin{array}{l}36 \cdot 9 \\
n\end{array}$ & $\begin{array}{l}1.6 \\
\%\end{array}$ & $\begin{array}{l}44 \cdot 1 \\
n\end{array}$ & $\begin{array}{l}3.8 \\
\%\end{array}$ & \\
\hline Area of residence & & & & & & & & & & & 0.098 \\
\hline Urban & 699 & 35.9 & 817 & $36 \cdot 1$ & 728 & 38.3 & 822 & $39 \cdot 1$ & 917 & 38.6 & \\
\hline Rural & 1155 & $59 \cdot 3$ & 1324 & 58.6 & 1053 & $55 \cdot 5$ & 1177 & $56 \cdot 0$ & 1331 & $56 \cdot 1$ & \\
\hline Remote & 93 & 4.8 & 119 & $5 \cdot 3$ & 118 & $6 \cdot 2$ & 101 & 4.8 & 125 & $5 \cdot 3$ & \\
\hline Marital status & & & & & & & & & & & 0.006 \\
\hline Married/de facto & 1463 & 74.9 & 1854 & $82 \cdot 0$ & 1578 & 83.0 & 1755 & $83 \cdot 6$ & 1991 & 84.0 & \\
\hline Separated/divorced & 331 & $16 \cdot 9$ & 289 & $12 \cdot 8$ & 204 & $10 \cdot 7$ & 230 & 11.0 & 246 & $10 \cdot 4$ & \\
\hline Widowed & 78 & 4.0 & 60 & $2 \cdot 7$ & 56 & 2.9 & 64 & 3.0 & 66 & $2 \cdot 8$ & \\
\hline Never married & 82 & 4.2 & 58 & $2 \cdot 6$ & 64 & 3.4 & 50 & $2 \cdot 4$ & 66 & $2 \cdot 8$ & \\
\hline Average household income & & & & & & & & & & & 0.001 \\
\hline Low & 1006 & 59.4 & 1056 & $52 \cdot 8$ & 872 & $51 \cdot 7$ & 893 & 48.5 & 1003 & $47 \cdot 0$ & \\
\hline Middle & 490 & 28.9 & 648 & 32.4 & 553 & $32 \cdot 8$ & 646 & $35 \cdot 1$ & 761 & 35.7 & \\
\hline High & 198 & $11 \cdot 7$ & 294 & $14 \cdot 7$ & 262 & $15 \cdot 5$ & 303 & $16 \cdot 4$ & 369 & $17 \cdot 3$ & \\
\hline Education & & & & & & & & & & & 0.001 \\
\hline No formal qualification & 1144 & 58.6 & 1158 & 51.4 & 899 & 47.4 & 907 & 43.4 & 960 & $40 \cdot 7$ & \\
\hline Higher school or trade certificate/diploma & 623 & 31.9 & 777 & 34.5 & 690 & $36 \cdot 4$ & 834 & 39.9 & 977 & $41 \cdot 3$ & \\
\hline University degree & 184 & 9.4 & 319 & $14 \cdot 2$ & 306 & $16 \cdot 1$ & 351 & $16 \cdot 8$ & 423 & $17 \cdot 9$ & \\
\hline Smoking status & & & & & & & & & & & 0.001 \\
\hline Never smoked/ex-smoker & 1521 & $78 \cdot 2$ & 1899 & $84 \cdot 0$ & 1642 & $86 \cdot 4$ & 1846 & $88 \cdot 1$ & 2153 & $90 \cdot 8$ & \\
\hline Light smoker & 86 & 4.4 & 117 & $5 \cdot 2$ & 87 & 4.6 & 97 & 4.6 & 108 & 4.6 & \\
\hline Heavy smoker & 339 & $17 \cdot 4$ & 244 & $10 \cdot 8$ & 171 & $9 \cdot 0$ & 153 & $7 \cdot 3$ & 109 & 4.6 & \\
\hline Physical activity & & & & & & & & & & & 0.001 \\
\hline None/sedentary & 568 & $31 \cdot 0$ & 468 & $21 \cdot 3$ & 309 & $16 \cdot 9$ & 255 & 12.5 & 213 & $9 \cdot 3$ & \\
\hline Low & 657 & 35.8 & 834 & 38.0 & 753 & $41 \cdot 2$ & 770 & 37.9 & 779 & 34.0 & \\
\hline Moderate & 277 & $15 \cdot 1$ & 418 & $19 \cdot 1$ & 365 & $20 \cdot 0$ & 451 & $22 \cdot 2$ & 552 & $24 \cdot 1$ & \\
\hline Heavy & 332 & $18 \cdot 1$ & 474 & $21 \cdot 6$ & 399 & 21.9 & 557 & $27 \cdot 4$ & 748 & $32 \cdot 6$ & \\
\hline Self-reported depression & 245 & $12 \cdot 6$ & 258 & 11.5 & 219 & 11.6 & 207 & 9.9 & 242 & $10 \cdot 3$ & 0.054 \\
\hline Use of antidepressants & 268 & $13 \cdot 7$ & 320 & $14 \cdot 1$ & 260 & 13.6 & 303 & 14.4 & 344 & 14.4 & 0.908 \\
\hline
\end{tabular}

* Number of participants varies for some variables because of missing data.

† Summarised by the Australian Recommended Food Score ${ }^{(21)}$. 
Table 2. Scores for each component of the Australian Recommended Food Score between 2001 and 2013 for women in the 1946-1951 cohort of the Australian Longitudinal Study on Women's Health ( $n$ 11 046) (Mean values and standard deviations; $\beta$ coefficients and $95 \%$ confidence intervals)

\begin{tabular}{|c|c|c|c|c|c|c|c|c|c|c|c|}
\hline & \multicolumn{8}{|c|}{ Diet quality scores } & & & \\
\hline & \multicolumn{2}{|c|}{2001} & \multicolumn{2}{|c|}{2007} & \multicolumn{2}{|c|}{2010} & \multicolumn{2}{|c|}{2013} & \multicolumn{3}{|c|}{ Diet quality change (units/year) } \\
\hline & Mean & SD & Mean & SD & Mean & SD & Mean & SD & $\beta$ & $95 \% \mathrm{Cl}$ & $P_{\text {trend }}$ \\
\hline Total & $32 \cdot 6$ & 8.8 & 32.5 & 8.8 & 31.9 & 8.5 & $33 \cdot 1$ & 8.6 & 0.01 & $-0.02,0.03$ & 0.089 \\
\hline \multicolumn{12}{|l|}{ Sub-components } \\
\hline Vegetables & $13 \cdot 6$ & 4.4 & $12 \cdot 2$ & 4.6 & $12 \cdot 0$ & 4.5 & $13 \cdot 8$ & 4.5 & -0.03 & $-0.04,-0.02$ & 0.001 \\
\hline Fruits & $5 \cdot 6$ & 3.2 & $5 \cdot 7$ & 3.2 & 5.4 & 3.0 & $5 \cdot 6$ & 3.1 & 0.01 & $-0.01,0.01$ & 0.842 \\
\hline Protein foods & $5 \cdot 2$ & 2.0 & $6 \cdot 0$ & $2 \cdot 0$ & $6 \cdot 0$ & 2.0 & 5.7 & 2.0 & 0.05 & $0.05,0.06$ & 0.001 \\
\hline Grains & 4.0 & 1.8 & 3.8 & 1.8 & 3.7 & 1.8 & 3.8 & 1.8 & -0.03 & $-0.03,-0.02$ & 0.001 \\
\hline Dairy products & $2 \cdot 1$ & 1.0 & 2.9 & 1.2 & $2 \cdot 9$ & $1 \cdot 1$ & $2 \cdot 2$ & 1.0 & 0.03 & $0.02,0.03$ & 0.001 \\
\hline Fats & 0.7 & 0.5 & 0.6 & 0.5 & 0.6 & 0.5 & 0.5 & 0.5 & -0.01 & $-0.01,-0.01$ & 0.001 \\
\hline Alcohol & 1.4 & 0.6 & 1.3 & 0.8 & 1.3 & 0.8 & 1.5 & 0.6 & 0.01 & $0.01,0.01$ & 0.001 \\
\hline
\end{tabular}

Table 3. Associations between diet quality* quintiles (Q) and depressive symptoms for women in the 1946-1951 cohort of the Australian Longitudinal Study on Women’s Health ( $n$ 11 046)† ( $\beta$ Coefficients and $95 \%$ confidence intervals)

\begin{tabular}{|c|c|c|c|c|c|c|c|c|c|c|}
\hline & \multirow[b]{2}{*}{$n \S$} & \multicolumn{2}{|c|}{ Q2 } & \multicolumn{2}{|c|}{ Q3 } & \multicolumn{2}{|c|}{ Q4 } & \multicolumn{2}{|c|}{ Q5 } & \multirow[b]{2}{*}{$P_{\text {trend }}$} \\
\hline & & $\beta$ & $95 \% \mathrm{Cl}$ & $\beta$ & $95 \% \mathrm{Cl}$ & $\beta$ & $95 \% \mathrm{Cl}$ & $\beta$ & $95 \% \mathrm{Cl}$ & \\
\hline Model 1 - univariate & 11046 & -0.31 & $-0.53,-0.09$ & -0.43 & $-0.65,-0.20$ & -0.43 & $-0.65,-0.20$ & -0.59 & $-0.81,-0.37$ & 0.001 \\
\hline Model 2 & 9122 & -0.12 & $-0.36,0.12$ & -0.24 & $-0.49,0.01$ & -0.19 & $-0.43,0.05$ & -0.36 & $-0.60,-0.11$ & 0.005 \\
\hline Model 3 & 8923 & -0.06 & $-0.30,0.19$ & -0.12 & $-0.37,0.14$ & -0.08 & $-0.32,0.18$ & -0.20 & $-0.45,0.05$ & 0.147 \\
\hline Model 4 & 8880 & -0.06 & $-0.31,0.15$ & -0.05 & $-0.29,0.19$ & -0.05 & $-0.29,0.18$ & -0.23 & $-0.47,0.01$ & 0.104 \\
\hline
\end{tabular}

* Summarised by the Australian Recommended Food Score ${ }^{(21)}$ as a time-varying variable.

† Model 1 was the univariate analysis including only diet quality and depressive symptoms. Model 2 was adjusted for indicators of socio-economic status: area of residence, marital status, average household income and education. Model 3 was adjusted as for model 2 and for smoking status and physical activity as time-varying variables. Model 4 was adjusted as for model 3 and self-reported physician diagnosis of depression and use of antidepressants. $\neq$ Depressive symptoms in each diet quality $Q$ compared with the lowest $Q$.

$\S$ Number of participants varies because of missing data for the covariates. 
Interestingly, as a result of the sensitivity analyses, we found that models including time-independent variables produced very different study estimates and significance levels compared with models including time-varying variables. This is an important finding as it justifies why our study findings differ from the previous two studies. The fact that previous studies are showing significant associations between diet quality and depression could be a result of not adequately accounting for variables that change over time, and thus are biased by residual confounding ${ }^{(31,32)}$. We believe that our approach of using a mixed model including time-varying variables provides a more valid and precise study estimate for the association of diet quality and depressive symptoms, as it accounts for the effects of time, and estimates change within each subject in addition to the average change in the study sample, while adjusting for time-varying confounding ${ }^{(33)}$.

The meta-analysis by our group showed that consumption of a healthy diet is associated with reduced odds of depression ${ }^{(5)}$. However, our meta-analysis only included four prospective cohort studies, and the subgroup analysis based on cohort studies suggests no significant association, although the study estimate was in the direction of an inverse association (OR 0.83; $95 \%$ CI 0.66, 1.05). More studies have been published since, arriving at a similar conclusion. One cohort study in Australia found no predictive effect of a prudent dietary pattern on depressive symptom incidence in middle-aged men and women ${ }^{(34)}$. The PREDIMED study randomised 7447 community-dwelling men and women aged 60-80 years at high risk of CVD to two Mediterranean diets and a low-fat diet (control group), and showed no significant difference in depression risk among participants assigned to a Mediterranean diet compared with the control group ${ }^{(35)}$. Note, however, that these studies focused on overall diet at predicting new cases of depression rather than reducing depressive symptoms among individuals with depressive disorder or sub-threshold depression.

Conversely, studies have demonstrated that unhealthy eating habits increased the likelihood of developing depression. In a French cohort study, high snacking habits were associated with elevated depressive symptoms ${ }^{(36)}$. The results from the Women's Health Initiative study suggest that high glycaemic index diets were associated with increased odds of depression $^{(37)}$. The Nurse's Health Study found a positive association between an inflammatory dietary pattern and depression ${ }^{(38)}$. These studies provided useful information regarding which unhealthy foods to avoid for a lower likelihood of developing depression, in addition to existing literature on healthy eating habits.

The present study is an extension of our previous investigation of diet quality and incident depression ${ }^{(14)}$, by examining the influence of diet quality on depressive symptoms, to explore the role of diet in effectively relieving depressive symptoms in individuals with depressive disorder or subthreshold depression. Our previous study found that maintaining good diet quality for at least 6 years has a borderline significance with lower odds of incident depression compared with maintaining poor diet quality ${ }^{(14)}$. The conflicting findings between the studies can be explained by the fact that the 
previous study made comparisons between extreme groups (i.e. long-term exposure to high- or low-quality diet), resulting in high between-subject variability in adherence, thus allowing an effect to be detected. That study also focused on a clinical diagnosis of depression, and it is possible that a dietary effect may not be detectable for sub-clinical depression or depressive symptoms.

The strength of the present study is that the data were drawn from a prospective study conducted among a large number of middle-aged community-dwelling women who are nationally representative of the Australian population. This study had a 12-year follow-up period with a high retention rate. We were able to carry out multiple assessments of diet quality and depressive symptoms using well-validated tools, and using the mixed-model approach allowed inclusion of subjects with incomplete data across time. All these contribute to increased statistical power.

Several limitations were noted as well. First, it is debatable whether the ARFS is the best tool to use to capture the aspect of diet most closely linked to depression. In fact, we are not certain of the ability of the ARFS at predicting other chronic diseases, as only one other study has used this score to predict type 2 diabetes, and that study showed no association between ARFS and diabetes, but found an association between diet quality - measured by the Dietary Guideline Index - and diabetes $^{(39)}$. To date, studies showing most consistent results with regard to diet quality score and chronic diseases are in relation to CVD risk because the diet quality score items were derived from epidemiological associations with reduced CVD risk and its risk factors ${ }^{(28)}$. Therefore, the null association in our study may be because the ARFS was not specifically designed based on current evidence for reduced depression risk. The lack of difference in diet quality over time may be a result of repeated assessments of diet using the same instruments, as participants may remember and repeat the same answers each time ${ }^{(40)}$. Although the sensitivity analyses showed similar performance in both ARFS and MDP index, the results are not directly comparable with our initial model (time-varying ARFS at four time points), and thus we cannot be sure of the ability of ARFS in predicting depression outcomes. Second, the CES-D measures depressive symptoms in the past 4 weeks. It is possible that participants completed the questionnaire when depressive symptoms were less severe (i.e. a form of healthy respondent bias). As such, the number of participants with depressive symptoms may be underestimated. Third, we included participants with self-reported physician diagnosis of depression and/or were using antidepressants, thus reverse causation is possible. However, we aimed to examine whether good diet quality relieves existing depressive symptoms and not its association with incident depression. In addition, including participants with existing depression diagnosis may confound the association but we have adjusted for these in our analysis. Although adjusting for these variables may contribute to unnecessary attenuation of the association, we have demonstrated that adjustment made little difference to the study estimates. Results may also be biased by unmeasured factors for example, dieting alters dietary intake and was associated with higher symptoms of emotional disorders ${ }^{(41)}$.

\section{Conclusion}

Our study results suggest no significant association between diet quality, assessed using ARFS, and depressive symptoms among middle-aged Australian women over a period of 12 years. Our results are concordant with randomised controlled trials and suggest that previous positive associations may have been due to residual confounding. It would be useful if similar analyses could be carried out using other diet quality indices to explore the association between diet quality and depressive symptoms to determine how robust the findings are. This study also highlights the need for more high-quality randomised controlled trials with longer follow-up time to definitely assess the role of diet in relieving existing depressive symptoms.

\section{Acknowledgements}

The research on which this paper is based was conducted as part of the Australian Longitudinal Study on Women's Health, the University of Newcastle and the University of Queensland. The authors also thank Professor Graham Giles of the Cancer Epidemiology Centre of Cancer Council Victoria for permission to use the Dietary Questionnaire for Epidemiological Studies (version 2), Melbourne: Cancer Council Victoria, 1996.

The authors are grateful to the Australian Government Department of Health who funded the study and the women who provided the survey data.

All authors were involved in the overall design and development of the research. J. S. L. conducted the research, which includes performing statistical analysis, writing the paper and had primary responsibility for the final content. C. O. assisted with statistical analysis. All the authors contributed to the editing of the paper and have read and approved the final version of the manuscript.

The authors declare that there are no conflicts of interest.

\section{Supplementary material}

For supplementary material/s referred to in this article, please visit http://dx.doi.org/doi:10.1017/S000711451500519X

\section{References}

1. Begg S, Vos T, Barker B, et al. (2007) The Burden of Disease and Injury in Australia 2003. Canberra: AIHW.

2. Fournier JC, DeRubeis RJ, Hollon SD, et al. (2010) Antidepressant drug effects and depression severity: a patientlevel meta-analysis. JAMA 303, 47-53.

3. van Zoonen K, Buntrock C, Ebert DD, et al. (2014) Preventing the onset of major depressive disorder: a meta-analytic review of psychological interventions. Int J Epidemiol 43, 318-329.

4. Commonwealth Department of Health and Aged Care \& Australian Institute of Health and Welfare (1999) NHPA Report on Mental Health 1998: A Report Focusing on Depression, Cat. no. PHE 11. Canberra: AIHW.

5. Lai JS, Hiles S, Bisquera A, et al. (2014) A systematic review and meta-analysis of dietary patterns and depression in community-dwelling adults. Am J Clin Nutr 99, 181-197.

6. Psaltopoulou T, Sergentanis TN, Panagiotakos DB, et al. (2013) Mediterranean diet, stroke, cognitive impairment, and depression: a meta-analysis. Ann Neurol 74, 580-591. 
7. McNaughton S (2010) Dietary patterns and diet quality: approaches to assessing complex exposures in nutrition. Australas Epidemiol 17, 35-37.

8. Michels KB \& Schulze MB (2005) Can dietary patterns help us detect diet-disease associations? Nutr Res Rev 18, 241-248.

9. Australian Bureau of Statistics (2014) Australian Health Survey: Nutrition First Results - Food and Nutrients, 2011-12, Cat. no. 4364.0.55.007. Canberra: ABS.

10. Flood VM, Burlutsky G, Webb KL, et al. (2010) Food and nutrient consumption trends in older Australians: a 10-year cohort study. Eur J Clin Nutr 64, 603-613.

11. Arabshahi S, Lahmann PH, Williams GM, et al. (2011) Longitudinal change in diet quality in Australian adults varies by demographic, socio-economic, and lifestyle characteristics. J Nutr 141, 1871-1879.

12. Australian Bureau of Statistics (2012) Australian Health Survey: First Results, 2011-12, Cat. no. 4364.0.55.001. Canberra: ABS

13. Haralambous B, Lin X, Dow B, et al. (2009) Depression in Older Age: A Scoping Study. Victoria: National Ageing Research Institute.

14. Lai J, Hure A, Oldmeadow C, et al. (2015) Prospective study on the association between diet quality and depression in mid-aged women over 9 years. Eur J Nutr (epublication ahead of print version 17 October 2015)

15. Ayuso-Mateos JL, Nuevo R, Verdes E, et al. (2010) From depressive symptoms to depressive disorders: the relevance of thresholds. Br J Psychiatry 196, 365-371.

16. Lee C, Dobson AJ, Brown WJ, et al. (2005) Cohort profile: the Australian Longitudinal Study on Women's Health. Int $J$ Epidemiol 34, 987-991.

17. Mishra GD, Loxton D, Anderson A, et al. (2014) Health and wellbeing of women aged 18 to 23 in 2013 and 1996: findings from the Australian Longitudinal Study on Women's Health. Report, Australian Government Department of Health.

18. Giles G \& Ireland P (1996) Dietary Questionnaire for Epidemiological Studies (Version 2). Melbourne: Cancer Council Victoria.

19. Hodge A, Patterson AJ, Brown WJ, et al. (2000) The anti cancer council of Victoria FFQ: relative validity of nutrient intakes compared with weighed food records in young to middle-aged women in a study of iron supplementation. Aust N Z J Public Health 24, 576-583.

20. Xinying PX, Noakes $M \&$ Keogh J (2004) Can a food frequency questionnaire be used to capture dietary intake data in a 4 week clinical intervention trial? Asia Pac J Clin Nutr 13, 318-323.

21. Collins CE, Young AF \& Hodge A (2008) Diet quality is associated with higher nutrient intake and self-rated health in mid-aged women. J Am Coll Nutr 27, 146-157.

22. Kant AK \& Thompson FE (1997) Measures of overall diet quality from a food frequency questionnaire: National Health Interview Survey, 1992. Nutr Res 17, 1443-1456.

23. National Health and Medical Research Council (2013) Australian Dietary Guidelines. Canberra: NHMRC.

24. Smith A, Kellett E \& Schmerlaib Y (1998) The Australian Guide to Healthy Eating. Canberra: Commonwealth Department of Health and Family Services.
25. Andresen EM, Malmgren JA, Carter WB, et al. (1994) Screening for depression in well older adults: evaluation of a short form of the CES-D (Center for Epidemiologic Studies Depression Scale). Am J Prev Med 10, 77-84.

26. Boey KW (1999) Cross-validation of a short form of the CES-D in Chinese elderly. Int J Geriatr Psychiatry 14, 608-617.

27. Trichopoulou A, Costacou T, Bamia C, et al. (2003) Adherence to a Mediterranean diet and survival in a Greek population. $N$ Engl J Med 348, 2599-2608.

28. Wirt A \& Collins CE (2009) Diet quality - what is it and does it matter? Public Health Nutr 12, 2473-2492.

29. Akbaraly TN, Sabia S, Shipley MJ, et al. (2013) Adherence to healthy dietary guidelines and future depressive symptoms: evidence for sex differentials in the Whitehall II study. Am J Clin Nutr 97, 419-427.

30. Sanchez-Villegas A, Delgado-Rodriguez M, Alonso A, et al. (2009) Association of the Mediterranean dietary pattern with the incidence of depression: the Seguimiento Universidad de Navarra/University of Navarra Follow-up (SUN) cohort. Arch Gen Psychiatry 66, 1090-1098.

31. Robins J (1986) A new approach to causal inference in mortality studies with a sustained exposure period - application to control of the healthy worker survivor effect. Math Model 7, 1393-1512.

32. Robins J (1987) A graphical approach to the identification and estimation of causal parameters in mortality studies with sustained exposure periods. J Chronic Dis 40, Suppl. 2, 139S-161S.

33. Gibbons RD, Hedeker D \& DuToit S (2010) Advances in analysis of longitudinal data. Annu Rev Clin Psychol 6, 79-107.

34. Jacka FN, Cherbuin N, Anstey KJ, et al. (2014) Dietary patterns and depressive symptoms over time: examining the relationships with socioeconomic position, health behaviours and cardiovascular risk. PLOS ONE 9, e87657.

35. Sanchez-Villegas A, Martinez-Gonzalez M, Estruch $\mathrm{R}$, et al. (2013) Mediterranean dietary pattern and depression: the PREDIMED randomized trial. BMC Med 11, 208.

36. Le Port A, Gueguen A, Kesse-Guyot E, et al. (2012) Association between dietary patterns and depressive symptoms over time: a 10-year follow-up study of the GAZEL cohort. PLOS ONE 7, e51593.

37. Gangwisch JE, Hale L, Garcia L, et al. (2015) High glycemic index diet as a risk factor for depression: analyses from the Women's Health Initiative. Am J Clin Nutr 102, 454-463.

38. Lucas M, Chocano-Bedoya P, Shulze MB, et al. (2014) Inflammatory dietary pattern and risk of depression among women. Brain Behav Immun 36, 46-53.

39. Alhazmi A, Stojanovski E, McEvoy M, et al. (2014) Diet quality score is a predictor of type 2 diabetes risk in women: the Australian Longitudinal Study on Women's Health. Br J Nutr 112, 945-951.

40. Jugenheimer DW, Kelley LD, Hudson J, et al. (2015) Advertising and Public Relations Research. New York: Taylor \& Francis.

41. Stunkard AJ \& Rush J (1974) Dieting and depression reexamined: a critical review of reports of untoward responses during weight reduction for obesity. Ann Intern Med 81, $526-533$. 